\title{
On the trade-off between energy efficiency and estimation error in compressive sensing is $^{2}$
}

\author{
Donglin $\mathrm{Hu}^{\mathrm{a}}$, Shiwen Mao ${ }^{\mathrm{a}, *}$, Nedret Billor ${ }^{\mathrm{b}}$, Prathima Agrawal ${ }^{\mathrm{a}}$ \\ ${ }^{a}$ Department of Electrical and Computer Engineering, Auburn University, Auburn, AL, USA \\ ${ }^{\mathrm{b}}$ Department of Mathematics and Statistics, Auburn University, Auburn, AL, USA
}

\section{A R T I C L E I N F O}

\section{Article history:}

Received 3 December 2012

Received in revised form 20 March 2013

Accepted 8 April 2013

Available online 28 April 2013

\section{Keywords:}

Bayesian estimation

Cognitive radio

Compressive sensing

Isotonic regression

Polynomial regression

Spectrum sensing

\begin{abstract}
A B S T R A C T
Compressive sensing (CS) refers to the process of reconstructing a signal that is supposed to be sparse or compressible. CS has wide applications, such as in cognitive radio networks. In this paper, we investigate effective CS schemes for the trade-off between energy efficiency and estimation error. We propose an enhancement to a Bayesian estimation approach and an enhancement to the isotonic regression approach that is based on nearly isotonic regression. We also show how to compute the routing matrix for selecting active sensor nodes. The proposed enhancements are evaluated with trace-driven simulations. Considerable gaps are observed between the original approaches and the proposed enhancements in the simulation results. The near isotonic regression method achieves the best performance among all the CS schemes examined in this paper.
\end{abstract}

(c) 2013 Elsevier B.V. All rights reserved.

\section{Introduction}

Compressive sensing (CS) (a.k.a. compressed sensing) refers to the process of reconstructing a signal that is supposed to be sparse or compressible [2]. It has found wide applications in communications and networking. For example, in cognitive radio networks, spectrum sensing is a critical component in dynamic spectrum access and enforcement of spectrum usage and sharing [3-7]. Given the wide range of activities in space, time, and frequency, it would be extremely challenging and costly to have a full range and dense sampling. In such situations, compressive sensing becomes a powerful tool for efficient spectrum sensing. Generally in a wireless sensor network (WSN), spatially distributed sensors are used to monitor physical

\footnotetext{
This paper was presented in part at IEEE GLOBECOM 2012, Anaheim, CA, USA, December 2012 [1]

* Corresponding author.

E-mail addresses: dzh0003@tigermail.auburn.edu (D. Hu), smao@ieee.org (S. Mao), billone@auburn.edu (N. Billor), agrawpr@auburn. edu (P. Agrawal).
}

or environmental conditions [8,9]. The sparse signals in wireless sensor networks are obtained by collecting readings from sensor nodes by a server (or, a data processing center) through wireless transmissions. The server will then use CS to process the sparse data to achieve certain design goals (e.g., recovering the missing data).

CS has received considerable interest from the wireless community recently. For example, several CS papers have focused on network optimization and scheduling, where two important factors, i.e., network performance and power consumption, are considered in the design of CS schemes. The objectives of the schemes presented in these papers are to prolong the life time of wireless sensor nodes, while meeting certain network performance requirements. Energy can be conserved by turning off some sensor nodes while keeping the rest active. Network performance can be measured in terms of surveillance quality such as the number of active nodes [10,11], network coverage [12], the minimum degree of connection $[13,14]$, and surveillance delay $[15,16]$. The proposed schemes usually aim to achieve a trade-off between network performance and power consumption. 
Other CS papers have laid emphasis on signal compression and reconstruction. These papers seek a trade-off between compression efficiency and reconstruction quality. For instance, the conventional CS approach is based on orthonormal basis. An important example of this approach is wavelet transform. The original sensor data is compressed and delivered [17]; therefore fewer network resource will be needed for transmitting the compressed data [18-20].

In this paper, we investigate effective CS schemes for addressing the fundamental trade-off between energy efficiency and estimation error. As discussed, a compressed signal can be obtained by collecting readings from a subset of the deployed sensor nodes, so that the rest of the nodes can be turned off to save energy. At the data processing center, the intact signal is reconstructed via signal estimation. Therefore, there is a fundamental trade-off between how many active nodes to choose (thus how much energy to spend) and the corresponding accuracy of prediction. Recently, only a few papers have investigated the problem of joint network optimization and signal processing. In [21], sensor nodes are divided into subgroups and all the readings can be recovered from one of the subgroups using isotonic regression. The objective is to maximize the number of subgroups while keeping the estimation error below a tolerable threshold. In [22], nodes are grouped into pairs. In each pair, the node with lower battery capacity goes to sleep, while the node with higher battery capacity estimates the measurement of the sleeping node using linear regression. In $[23,24]$, the authors investigate the problem of reconstructing a distributed signal through the collection of a small number of sensor readings, where CS is applied in conjunction with principle component analysis. The data of the entire network is recovered with Bayesian estimation.

In particular, we aim to develop effective CS schemes in WSNs and seek to balance the trade-off between energy saving and recovery accuracy, which are in terms of the number of active sensor nodes and the corresponding estimation error. We examine three classes of CS approaches in related work discussed above. We first implement the Bayesian estimation approach presented in [23], and propose an enhancement to this approach by scaling the variance of the sensor readings. We next revisit the isotonic regression approach presented in [21], and propose an improved method based on nearly isotonic regression. Finally, we briefly introduce two polynomial regression approaches, i.e., linear regression and quadratic regression, which are used as benchmarks in the performance evaluation. Bayesian estimation selects active nodes randomly, while isotonic regression chooses active nodes based on estimation errors, which results in better reconstruction quality. Unlike LR and QR, isotonic regression considers the distribution of readings for more accurate estimation. The proposed enhancements are evaluated with trace-driven simulations. We find considerable gaps between the original approaches and the proposed enhancements in the simulation results. We also find that the near isotonic regression method achieves the best performance among all the CS schemes examined in this paper.

The remainder of this paper is organized as follows. In Section 2, we describe the system model and assumptions.
We introduce Masiero's method and its enhancement in Section 3. In Section 4.1, we discuss Koushanfar's method and the nearly isotonic regression based enhancement. In Section 5, we present two polynomial regression schemes. We evaluate the proposed enhancements with trace-driven simulations in Section 6. Section 7 concludes this paper.

\section{System model and assumptions}

The network model considered in this paper consists of autonomous sensor nodes that are deployed in an area to monitor physical conditions (e.g., spectrum availability) and a data processing center (or, a server), as illustrated in Fig. 1. We assume that the sensor nodes collect information data following a synchronized slot structure. Once a node obtains its data in a time slot, it transmits the data to the data processing center through its wireless interface in the same time slot.

We assume the process of sensing and transmission of one unit of sensor data consumes a certain amount of energy, denoted as $E$, which is a constant. If there are $N$ sensor nodes deployed, the total amount of energy consumed in each time slot is $N \times E$. It is easy to see that a convenient way to conserve energy for the WSN is to reduce the number of active sensor nodes. On the other hand, the data from the idle nodes has to be recovered through CS to ensure certain precision of detection. The estimation errors of the recovered data should meet the minimum precision requirement of the target sensing application. Therefore, there is a fundamental trade-off between the number of active nodes and estimation error.

In the following sections, we introduce three classes of CS approaches and propose enhanced algorithms. The first class is Bayesian Estimation approaches, which focus on energy saving by managing the number of active sensor nodes. Specifically, $L$ active sensor nodes will be chosen from the set of $N$ sensor nodes to collect sensing data in each time slot. The second class includes the Isotonic Regression approaches and the third class is Polynomial Regression approaches, which guarantee the quality of recovered data by controlling the estimation error. With these techniques, we choose as few active sensor nodes

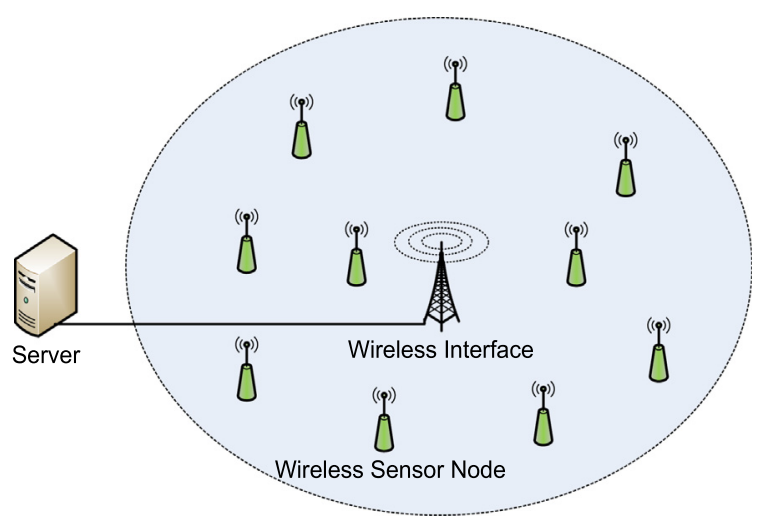

Fig. 1. The wireless sensor network model. 
as possible, while keeping the estimation error below a prescribed threshold. The notations used in this paper and their definitions are summarized in Table 1.

\section{Bayesian estimation approaches}

In Bayesian Estimation (BE), the parameter set $\theta$ is obtained by maximizing the posterior probability density function (pdf)

$p(\theta \mid \mathcal{D}, \mathcal{M})=\frac{p(\mathcal{D} \mid \theta, \mathcal{M}) p(\theta \mid \mathcal{M})}{p(\mathcal{D} \mid \mathcal{M})}$,

where $\mathcal{D}$ is the data set and $\mathcal{M}$ the plausible model. Since $p(\mathcal{D} \mid \mathcal{M})$ does not include $\theta$, maximizing the posterior probability $p(\theta \mid \mathcal{D}, \mathcal{M})$ is equivalent to maximizing the product of $p(\mathcal{D} \mid \theta, \mathcal{M})$ and $p(\theta \mid \mathcal{M})$.

In [24], Masiero et al. propose an approach in which Principal Component Analysis (PCA) and CS are jointly considered. In the following, we first introduce this method in Section 3.1 for the sake of completeness. We then propose an enhanced joint PCA and CS method in Section 3.2.

Table 1

Notation

\begin{tabular}{|c|c|}
\hline Symbol & Definition \\
\hline $\mathcal{D}$ & The data set \\
\hline$\theta$ & The sensor parameter set \\
\hline $\mathcal{M}$ & The plausible model \\
\hline$N$ & The total number of sensor nodes \\
\hline$L$ & The number of active nodes \\
\hline$K$ & The number of sampling times \\
\hline E & $\begin{array}{l}\text { Energy consumption for sensing and } \\
\text { transmission of one unit of data }\end{array}$ \\
\hline$s_{X}$ & A sensor node $X$ in the network \\
\hline$s_{Y}$ & A sensor node $Y$ in the network \\
\hline$x_{k}$ & A reading from sensor $s_{X}$ at time $k$ \\
\hline$y_{k}$ & A reading from sensor $s_{Y}$ at time $k$ \\
\hline$\hat{y}_{k}$ & An estimate of $y_{k}$ \\
\hline $\mathbf{x}_{k}$ & The readings from all sensor nodes at time $k$ \\
\hline$\hat{\mathbf{x}}_{k}$ & The estimate of $\mathbf{x}_{k}$ \\
\hline $\mathbf{x}_{k}^{\prime}$ & The readings from active nodes at time $k$ \\
\hline $\mathbf{s}_{k}$ & The principal component of $\mathbf{x}_{k}$ \\
\hline$s_{k}^{i}$ & The $i$ th element of $\mathbf{s}_{k}$ \\
\hline $\mathbf{z}_{k}$ & A known data set at time $k$ \\
\hline$\overline{\mathbf{x}}$ & $\begin{array}{l}\text { The sample mean vector of } \mathbf{x}_{k} \text { over } \\
\text { time } k\end{array}$ \\
\hline$\beta$ & A weighted least-square fit to a vector $\mathbf{y}$ \\
\hline$\hat{\boldsymbol{\beta}}$ & The optimal solution to (16) \\
\hline g & The weight vector \\
\hline$\Sigma$ & $\begin{array}{l}\text { The sample covariance matrix of } \mathbf{x}_{k} \text { over } \\
\text { time } k\end{array}$ \\
\hline$\lambda_{i}$ & The $i$ th eigenvalue of $\Sigma$ \\
\hline $\mathbf{U}$ & $\begin{array}{l}\text { Orthonormal matrix consisting of } \\
\text { eigenvectors of } \Sigma\end{array}$ \\
\hline $\mathcal{G}$ & The Gaussian distribution assumption \\
\hline $\mathcal{L}$ & The Laplace distribution assumption \\
\hline$\Phi$ & The routing matrix \\
\hline$\epsilon_{X, Y}$ & $\begin{array}{l}\text { The estimation error of } s_{Y} \text { based on the } \\
\text { readings of } s_{X}\end{array}$ \\
\hline $\mathbf{R}$ & The relative importance matrix \\
\hline $\mathbf{E}$ & The error matrix \\
\hline C & The cumulative error matrix \\
\hline
\end{tabular}

\subsection{Joint PCA and CS}

In the network, the readings of sensor nodes, e.g., the illumination of an outdoor environment, are collected with a fixed sampling rate at discrete times $k=1,2, \cdots, K$. Let $\mathbf{x}_{k} \in \mathbb{R}^{N}$ be an $N \times 1$ vector that contains the measurements collected at time $k$. Then $\mathbf{x}_{k}$ can be considered as an $N$ dimensional stochastic process.

With PCA, the centered $\mathbf{x}_{k}$ can be projected to a new $N \times 1$ variable $\mathbf{s}_{k}$, which is written as

$\mathbf{s}_{k}=\mathbf{U}^{T} \cdot\left(\mathbf{x}_{k}-\overline{\mathbf{x}}\right)$,

where $\overline{\mathbf{x}}$ is the mean of $\mathbf{x}$ and $\mathbf{U}$ is an orthonormal matrix whose column vectors are the eigenvectors of the covariance matrix of $\mathbf{x}_{k}$. The eigenvectors are placed in the decreasing order of the corresponding eigenvalues $\left(\lambda_{i}\right)$ The mean vector and covariance matrix can be replaced by the sample mean vector and the sample covariance matrix as

$\overline{\mathbf{x}}=\frac{1}{K} \sum_{k=1}^{K} \mathbf{x}_{k}$,

$\Sigma=\frac{1}{K} \sum_{k=1}^{K}\left(\mathbf{x}_{k}-\overline{\mathbf{x}}\right)\left(\mathbf{x}_{k}-\overline{\mathbf{x}}\right)^{T}$.

According to the properties of PCA, it can be shown that $s_{k}^{i}$, the $i$ th element of $\mathbf{s}_{k}$, is uncorrelated with $s_{k}^{j}$. That is,

$\mathbb{E}\left[s_{k}^{i} s_{k}^{j}\right]=\mathbb{E}\left[s_{k}^{i}\right] \mathbb{E}\left[s_{k}^{j}\right]=0, \quad \forall i \neq j$.

Furthermore, the elements of $\mathbf{s}_{k}$ are independent of each other if they follow the Gaussian distribution. The joint probability density function (pdf) of $\mathbf{s}_{k}$ can be decomposed into the product of the individual pdfs of its elements. In CS, not all the elements of $\mathbf{x}_{k}$ can be obtained at time $k$ because a portion of sensor nodes are turned off to reduce power consumption. Similarly, we only have partial elements of $\mathbf{s}_{k}$ from the projection of the partial elements of the centered $\mathbf{x}_{k}$. Assume there are $L$ active nodes in the WSN. Let $\mathbf{x}_{k}^{\prime}$ be an $L \times 1$ vector that collects measurements from the $L$ active nodes. The $L$ active nodes are selected randomly from the $N$ sensor nodes with an $L \times N$ routing matrix $\boldsymbol{\Phi}$, which is a binary matrix comprised of two values 0 and 1 . Each row of $\boldsymbol{\Phi}$ has only a single 1 element whose position corresponds to the index of the selected sensor node.

The relationship between $\mathbf{x}_{k}^{\prime}$ and $\mathbf{x}_{k}$ can be written as $\mathbf{x}_{k}^{\prime}=\boldsymbol{\Phi} \cdot \mathbf{x}_{k}$.

Let $\mathbf{z}_{k}=\mathbf{x}_{k}^{\prime}-\boldsymbol{\Phi} \cdot \overline{\mathbf{x}}$. Recall that $\mathbf{x}_{k}^{\prime}$ is a vector containing the measurements from active nodes and $\overline{\mathbf{x}}$ is the sample mean vector. Both of them are available at time $k$. Thus, $\mathbf{z}_{k}$ is a known data set at time $k$. By combining Eqs. (2) and (6), $\mathbf{z}_{k}$ can also be obtained from $\mathbf{s}_{k}$, as

$\mathbf{z}_{k}=\boldsymbol{\Phi}\left(\mathbf{x}_{k}-\overline{\mathbf{x}}\right)=\boldsymbol{\Phi} \cdot \mathbf{U} \mathbf{s}_{k}$

The objective of this method is to estimate the parameter set $\mathbf{s}_{k}$ from data set $\mathbf{z}_{k}$. The estimate of the parameter set $\mathbf{s}_{k}$ is denoted as $\hat{\mathbf{s}}_{k}$. Once $\hat{\mathbf{s}}_{k}$ is obtained, the intact signal $\mathbf{x}_{k}$ can be recovered from $\hat{\mathbf{s}}_{k}$ according to (2), as

$\hat{\mathbf{x}}_{k}=\overline{\mathbf{x}}+\mathbf{U} \cdot \hat{\mathbf{s}}_{k}$, 
where $\hat{\mathbf{x}}_{k}$ is the estimate of $\mathbf{x}_{k}$. Two types of distribution assumptions of $\mathbf{s}_{k}$ were made in [23], i.e., Gaussian distribution and Laplace distribution. They are good models to represent the principal components of typical sensing data. Interested readers are referred to [23] for details.

\subsubsection{Gaussian distribution}

The authors first assume the elements of $\mathbf{s}_{k}$ follow Gaussian distribution, denoted as $\mathcal{N}\left(\mu_{0}, \sigma_{0}^{2}\right)$. Since $s_{k}^{i}$ 's are uncorrelated, they are independent of each other due to the Gaussian assumption. Therefore $p(\theta \mid \mathcal{M})$, the prior probability, can be computed as:

$$
\begin{aligned}
p(\theta \mid \mathcal{M}) & =p\left(\mathbf{s}_{k} \mid \mathcal{N}\right)=\prod_{i=1}^{N} \frac{1}{\sqrt{2 \pi \sigma_{0}^{2}}} \exp \left\{-\frac{\left(s_{k}^{i}-\mu_{0}\right)^{2}}{2 \sigma_{0}^{2}}\right\} \\
& =\frac{1}{\left(2 \pi \sigma_{0}^{2}\right)^{\frac{N}{2}}} \exp \left\{-\frac{\sum_{i=1}^{N}\left(s_{k}^{i}-\mu_{0}\right)^{2}}{2 \sigma_{0}^{2}}\right\} .
\end{aligned}
$$

The mean $\mu_{0}$ and variance $\sigma_{0}^{2}$ of the Gaussian distribution can be estimated from the past $\mathbf{s}_{l}(l<k)$. According to (7), the relationship between $\mathbf{z}_{k}$ and $\mathbf{s}_{k}$ can be translated into a likelihood of the form:

$p(\mathcal{D} \mid \theta, \mathcal{M})=p\left(\mathbf{z}_{k} \mid \mathbf{s}_{k}, \mathcal{N}\right)=\delta\left(\mathbf{z}_{k}, \boldsymbol{\Phi} \mathbf{U}_{k} \mathbf{s}_{k}\right)$,

where $\delta(x, y)$ is 1 if $x=y$ and 0 otherwise. As discussed, the parameter set $\mathbf{s}_{k}$ is estimated by maximizing posterior probability density, which can be written as:

$$
\begin{aligned}
\hat{\mathbf{s}}_{k} & =\operatorname{argmax} p(\theta \mid \mathcal{D}, \mathcal{M})=\operatorname{argmax} p(\theta \mid \mathcal{M}) p(\mathcal{D} \mid \theta, \mathcal{M}) \\
& =\operatorname{argmax} \frac{\delta\left(\mathbf{z}_{k}, \boldsymbol{\Phi} \mathbf{U}_{k} \mathbf{s}_{k}\right)}{\left(2 \pi \sigma_{0}^{2}\right)^{\frac{N}{2}}} \exp \left\{-\frac{\sum_{i=1}^{N}\left(s_{k}^{i}-\mu_{0}\right)^{2}}{2 \sigma_{0}^{2}}\right\} \\
& =\operatorname{argmin} \sum_{i=1}^{N}\left(s_{k}^{i}-\mu_{0}\right)^{2}, \quad \text { given that } \mathbf{z}_{k}=\boldsymbol{\Phi} \mathbf{U}_{k} \mathbf{s}_{k} .
\end{aligned}
$$

Problem (11) is a typical convex problem with linear equality constraints. It can be solved with some mathematical packages such as CVX $[25,26]$.

\subsubsection{Laplace distribution}

The authors also assume $\mathbf{s}_{k}$ follow Laplace distribution $\mathcal{L}\left(\mu_{1}, \sigma_{1}\right)$, where $\mu_{1}$ is a location parameter and $\sigma_{1}$ is a scale parameter. Laplace distribution with zero mean is widely used in the literature [24] to statistically model sparse random vectors. Similarly, it follows that

$$
\begin{aligned}
\hat{\mathbf{s}}_{k} & =\arg \max p\left(\mathbf{s}_{k} \mid \mathbf{z}_{k}, \mathcal{L}\right) \\
& =\operatorname{argmax} \frac{\delta\left(\mathbf{z}_{k}, \boldsymbol{\Phi} \mathbf{U}_{k} \mathbf{s}_{k}\right)}{\left(2 \sigma_{1}^{2}\right)^{\frac{N}{2}}} \exp \left\{-\frac{\sum_{i=1}^{N}\left|s_{k}^{i}-\mu_{1}\right|}{\sigma_{1}}\right\} \\
& =\operatorname{argmin} \sum_{i=1}^{N}\left|s_{k}^{i}-\mu_{1}\right|, \quad \text { given that } \mathbf{z}_{k}=\boldsymbol{\Phi} \mathbf{U}_{k} \mathbf{s}_{k},
\end{aligned}
$$

where $\mu_{1}$ is estimated using the median of $\mathbf{s}_{l}(l<k)$. Once $\hat{\mathbf{s}}_{k}$ is obtained, $\mathbf{x}_{k}$ can be recovered as shown in (8).

\subsection{Enhanced PCA-CS scheme}

The major disadvantage of the above approach is that the difference in the variances of the principal components is not considered. It is assumed that the elements of $\mathbf{s}_{k}$ follow the same distribution with identical variance. However, we show in the following proposition that the above assumption does not generally hold true.

Proposition 1. For any given pair of eigenvalues $\lambda_{i}>\lambda_{j}$, the corresponding variance ofs $s_{k}^{i}$ is greater than that ofs ${ }_{k}^{j}$, i.e., $\mathbb{E}\left[\left(s_{k}^{i}\right)^{2}\right]>\mathbb{E}\left[\left(s_{k}^{j}\right)^{2}\right]$ if $\lambda_{i}>\lambda_{j}$.

Proof. From (2), it follows that

$$
\begin{aligned}
\mathbb{E}\left[\mathbf{S}_{k} \mathbf{s}_{k}^{T}\right] & =\mathbf{U}^{T} \mathbb{E}\left[\left(\mathbf{x}_{k}-\overline{\mathbf{x}}\right)\left(\mathbf{x}_{k}-\overline{\mathbf{x}}\right)^{T}\right] \mathbf{U}=\mathbf{U}^{T} \Sigma \mathbf{U} \\
& =\operatorname{diag}\left(\lambda_{1}, \ldots, \lambda_{N}\right),
\end{aligned}
$$

where $\operatorname{diag}\left(\lambda_{1}, \cdots, \lambda_{N}\right)$ is a diagonal matrix with diagonal elements $\left(\lambda_{1}, \cdots, \lambda_{N}\right)$. Then we have $\mathbb{E}\left[\left(s_{k}^{i}\right)^{2}\right]=\lambda_{k}$ and the statement follows.

To remove this generally unrealistic assumption, the main idea of our enhanced method is to scale $s_{i}^{k}$ according to its corresponding variance $\lambda_{i}$. We substitute $\mathbf{U}$ with another matrix $\mathbf{V}=\mathbf{U} \Lambda$ where

$\Lambda=\operatorname{diag}\left(1 / \sqrt{\lambda_{1}}, 1 / \sqrt{\lambda_{2}}, \cdots, 1 / \sqrt{\lambda_{N}}\right)$,

is a diagonal matrix. Obviously $\mathbf{V}$ is also an orthonormal matrix by definition. Defining

$\mathbf{d}_{k} \stackrel{\text { def }}{=} \mathbf{V}^{T}\left(\mathbf{x}_{k}-\overline{\mathbf{x}}\right)$,

it follows that

$$
\begin{aligned}
\mathbb{E}\left[\mathbf{d}_{k} \mathbf{d}_{k}^{T}\right] & =\mathbf{V}^{T} \mathbb{E}\left[\left(\mathbf{x}_{k}-\overline{\mathbf{x}}\right)\left(\mathbf{x}_{k}-\overline{\mathbf{x}}\right)^{T}\right] \mathbf{V}=\Lambda^{T} \mathbf{U}^{T} \Sigma \mathbf{U} \Lambda \\
& =\Lambda^{T} \operatorname{diag}\left(\lambda_{1}, \ldots, \lambda_{N}\right) \Lambda=\mathbf{I}
\end{aligned}
$$

where $\mathbf{I}$ is the $N \times N$ identity matrix. In our proposed algorithm, we replace matrix $\mathbf{U}$ with the new matrix $\mathbf{V}$. As a result, the elements of the new projected vector $\mathbf{d}_{k}$ will have identical variance.

The two distribution assumptions used in [23] (described in Sections 3.1.1 and 3.1.2) can also be adopted for the enhanced method. In both models, the elements are assumed to follow the same distribution. Since the elements of $\mathbf{d}_{k}$ now have identical variance, it fits the model assumption much better than $\mathbf{s}_{k}$ and consequently, the accuracy of recovered data will be improved, as will be shown in our simulation study.

\section{Isotonic regression approaches}

With Bayesian Estimation, we are able to manage $L$, the number of active nodes, and the percentage of power saved is computed as $(N-L) / N$. However, there is no consideration of the distance between the true measurements $\mathbf{x}_{k}$ and the recovered measurements $\hat{\mathbf{x}}_{k}$. In many applications, the quality of reconstruction is no less important than energy saving. In this section, we introduce Isotonic Regression (IR) that is able to address this problem.

Isotonic regression aims to find a weighted least-square fit $\boldsymbol{\beta} \in \mathbb{R}^{n}$ to a vector $\mathbf{y} \in \mathbb{R}^{n}$ with weight vector $\mathbf{g} \in \mathbb{R}^{n}$, sub- 
ject to a set of monotonicity constraints. The IR problem is defined as follows:

minimize : $\sum_{i=1}^{n} g_{i}\left(y_{i}-\beta_{i}\right)^{2}$

subject to : $\beta_{1} \leqslant \cdots \leqslant \beta_{n}$,

where $y_{i}, g_{i}$ and $\beta_{i}$ are the $i$ th element of vectors $\mathbf{y}, \mathbf{g}$ and $\boldsymbol{\beta}$, respectively. The optimal solution $\hat{\boldsymbol{\beta}}$ is a function of $\mathbf{y}$.

This is motivated by the fact that the readings of neighboring sensor nodes are usually positively correlated. Suppose two sensors $s_{X}$ and $s_{Y}$ are exposed to the same stimuli source. Intuitively, increasing the stimuli value may result in higher readings at both sensors. It is thus possible to predict the reading of $s_{Y}$ from the reading of $s_{X}$, and vice versa.

\subsection{Combinatorial isotonic regression}

In [21], the authors propose a method, named Combinatorial Isotonic Regression (CIR), for solving the isotonic regression problem. A four-step method is presented. The first two steps are used to convert isotonic regression into a combinatorial problem. Dynamic programming is then used to solve the combinatorial problem.

Denote the time series of reading from two sensors as $\mathbf{X}=\left\{x_{k}\right\}$ and $\mathbf{Y}=\left\{y_{k}\right\}, k=1,2, \cdots, K$. CIR sorts the values of $\mathbf{X}$ and $\mathbf{Y}$ in strictly increasing order and eliminates redundancies by grouping the identical values. We then obtain $x_{(1)}$ $<\cdots<x_{(i)}<\cdots<x_{(n)}$ and $y_{(1)}<\cdots<y_{(j)}<\cdots<y_{(m)}$. The objective is to find a mapping $\mathbf{X} \rightarrow \mathbf{Y}$ by solving

minimize : $\sum_{i=1}^{n} \sum_{j=1}^{m} g_{i, j}\left(y_{(j)}-\beta_{i}\right)^{2}$

subject to : $\beta_{1} \leqslant \cdots \leqslant \beta_{n}$,

where $g_{i, j}$ is the number of $\left\{x_{(i)}, y_{(j)}\right\}$ pairs of readings from sensor $s_{X}$ and $s_{Y}$. The optimal solution $\hat{\beta}_{i}$ is an estimate of $y_{k}$ when reading $x_{k}$ at time $k$ is equal to $x_{(i)}$. Note that the L-1 norm is used in [21] and their method also works in the case of L-2 norm. To be consistent with the other methods, we adopt the L-2 norm in the remainder of this paper.

The CIR method consists of four steps, which are executed for each pair of sensor nodes. The algorithm is given below.

Step 1 : Build the relative importance matrix $\mathbf{R}$, which captures the number of the pairs $\left\{x_{(i)}, y_{(j)}\right\}$.

Step 2 : Build the error matrix $\mathbf{E}$ whose element $\left\{e_{i, j}\right\}$ describes the error that would be made if the prediction of $\mathbf{Y}$ was chosen $y_{(j)}$ while the reading $x_{(i)}$ was obtained from $s_{X}$. The error element $e_{i, j}$ is defined as follows:

$$
\begin{aligned}
e_{i, j} & =\sum_{l=1}^{m} r_{i, j} \times\left(y_{(l)}-y_{(j)}\right)^{2} \text { for } i=1, \cdots, n ; \\
j & =1, \cdots, m
\end{aligned}
$$

where $r_{i, j}$ is the element of matrix $\mathbf{R}$ in the $i$ th row and $j$ th column. Recall that $m$ and $n$ are the number of grouped $x_{k}$ and the number of grouped $y_{k}$, respectively.

Step 3 : Build a cumulative error matrix C. Each element $c_{i, j}$ of the matrix $\mathbf{C}$ describes the accumulated errors from left column to right. CIR computes $c_{i, j}$ as follows:

$$
c_{i, j}= \begin{cases}e_{i, j}, & \text { if } j=1, \\ e_{i, j}+\min _{1 \leqslant i^{\prime} \leqslant i}\left\{c_{i^{\prime}, j-1}\right\}, & \text { otherwise. }\end{cases}
$$

For each value in $\mathbf{C}$, keep the index

$$
d_{i, j}=\underset{1 \leqslant i^{\prime} \leqslant i}{\operatorname{argmin}}\left\{c_{i^{\prime}, j-1}\right\},
$$

that tracks the minimum value in its previous column, which leads to the current estimate of the cumulative error.

Step 4 : Once the cumulative matrix $\mathbf{C}$ is obtained, find the minimum value in the last column of $\mathbf{C}$ and the path leading to this value by back tracking the indices.

Steps 3 and 4 are presented in Algorithm 1. The complexity of this algorithm is $\mathcal{O}\left(\mathrm{nm}^{2}\right)$.

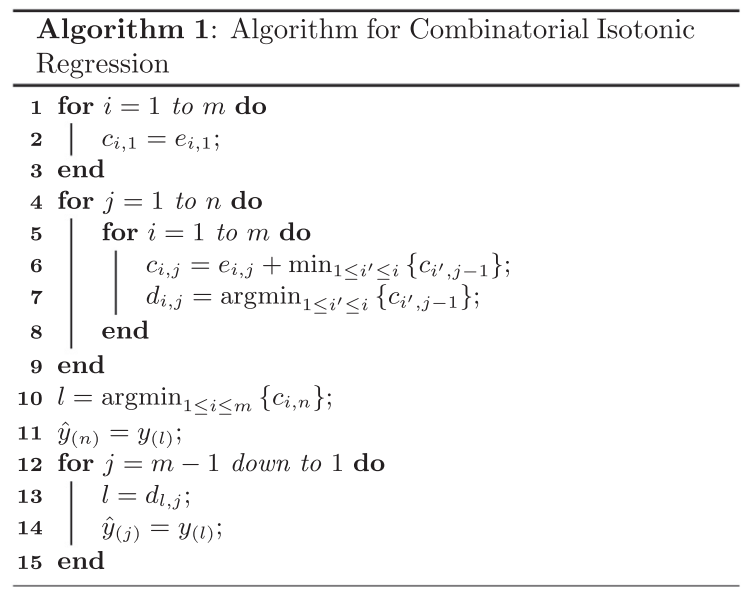

\subsection{Nearly isotonic regression}

CIR is based on dynamic programming to find the optimal value of objective function [21]. However, in Step 2, it can be seen that the estimate of the reading from sensor $s_{Y}$ can only be selected from the set of $y_{(j)}$ 's. To improve the CIR method, we propose a Nearly Isotonic Regression (NIR) based method in this section.

In particular, We adopt a method originally proposed for solving NIR problems in [27], to estimate the reading of $s_{Y}$ from that of $s_{X}$. Again, we group the same value of $\mathbf{X}$ and average the values of readings at $s_{Y}$ when $\mathbf{X}$ takes value $x_{(i)}$. Denote $\bar{y}_{i}$ as the average reading at $s_{Y}$ when $\mathbf{X}$ takes $x_{(i)}$. The problem can be formulated as: 
minimize : $\sum_{i=1}^{n}\left(\bar{y}_{i}-\beta_{i}\right)^{2}$

subject to : $\beta_{1} \leqslant \cdots \leqslant \beta_{n}$.

The optimal solution $\hat{\beta}_{i}$ of this problem is an estimate of $y_{k}$ when reading $x_{k}$ is $x_{(i)}$ at sensor $s_{X}$. After obtaining $\hat{\beta}_{i}$, the reading from sensor $s_{X}$ is used to estimate that of sensor $s_{Y}$. If the reading of $s_{X}$ is $x_{(i)}$, the estimate of the reading of $s_{Y}$ is $\beta_{i}$.

In [27], Tibshirani et al. seek a nearly-monotone approximation and considers the following problem:

$\hat{\beta}_{\lambda}=\underset{\beta_{i}}{\operatorname{argmin}}\left\{\frac{1}{2} \sum_{i=1}^{n}\left(\bar{y}_{i}-\beta_{i}\right)^{2}+\lambda \sum_{i=1}^{n-1}\left(\beta_{i}-\beta_{i+1}\right)_{+}\right\}$,

where $x_{+}$indicates the positive part of $x$, i.e., $x_{+}=x \cdot 1_{[x>0]}$. This is a convex problem for each fixed $\lambda \geqslant 0$. We have Proposition 2 from [27] to solve this problem.

Proposition 2. Suppose that, for some $\lambda$, two adjacent coordinates of the solution satisfy $\hat{\beta}_{\lambda, i}=\hat{\beta}_{\lambda, i+1}$. Then we have $\hat{\beta}_{\lambda_{0}, i}=\hat{\beta}_{\lambda_{0}, i+1}$, for all $\lambda_{0} \geqslant \lambda[27]$.

Proposition 2 states that the pieces in the solution can only be joined together, not split apart, as $\lambda$ increases. Consider a parameter value $\lambda$ with $K_{\lambda}$ joined pieces, which are represented by groups of coordinates $A_{1}, \ldots, A_{K_{\lambda}}$. Problem (21) can be rewritten as

$\frac{1}{2} \sum_{i=1}^{K_{\lambda}} \sum_{j \in A_{i}}\left(\bar{y}_{j}-\beta_{\lambda, A_{i}}\right)^{2}+\lambda \sum_{i=1}^{K_{\lambda}-1}\left(\beta_{\lambda, A_{i}}-\beta_{\lambda, A_{i+1}}\right)_{+} \cdots$

To find the optimal values $\hat{\beta}_{\lambda, A_{i}}$, we examine the subgradient of (22), as

$$
\begin{aligned}
& -\sum_{j \in A_{i}} \bar{y}_{j}+\left|A_{i}\right| \hat{\beta}_{\lambda, A_{i}}+\lambda\left(w_{i}-w_{i-1}\right)=0 \text { for } i \\
& \quad=1, \ldots, K_{\lambda},
\end{aligned}
$$

where $w_{i}=1_{\left[\hat{\beta}_{\lambda, A_{i}}-\hat{\beta}_{\lambda, A_{i+1}}>0\right]}$ and $w_{0}=w_{K_{\lambda}}=0$. We then differentiate (23) with respect to $\lambda$ to get the slope $m_{i}$ as

$m_{i}=\frac{d \hat{\beta}_{\lambda, A_{i}}}{d \lambda}=\frac{w_{i-1}-w_{i}}{\left|A_{i}\right|}$

The slope $m_{i}$ is a constant, indicating that each $\hat{\beta}_{\lambda, A_{i}}$ is a linear function of $\lambda$.

Using this slope, it can be shown that groups $A_{i}$ and $A_{i+1}$ will merge at

$t_{i, i+1}=\frac{b_{i+1}-b_{i}}{m_{i}-m_{i+1}}, \quad$ for $i=1, \ldots, K_{\lambda}-1$,

where $b_{i}=\frac{\sum_{j \in A_{i}} \bar{y}_{j}}{\left|A_{i}\right|}$. We can move to the next value of $\lambda$

$\lambda^{*}=\min _{i: t_{i, i+1}>\lambda}\left\{t_{i, i+1}\right\}$,

and merge groups $A_{i^{*}}$ and $A_{i^{*}+1}$, where

$i^{*}=\underset{i: t_{i, i+1}>\lambda}{\operatorname{argmin}}\left\{t_{i, i+1}\right\}$.

Note that the minimization problems (26) and (27) are only taken over the values of $t_{i, i+1}$ that are larger than $\lambda$.
If none of the $t_{i, i+1}$ 's are larger than $\lambda$, then none of the existing groups will merge and the algorithm will be terminated. The enhancement algorithm is presented in Algorithm 2. The worst case complexity of this algorithm is $O(n)$.

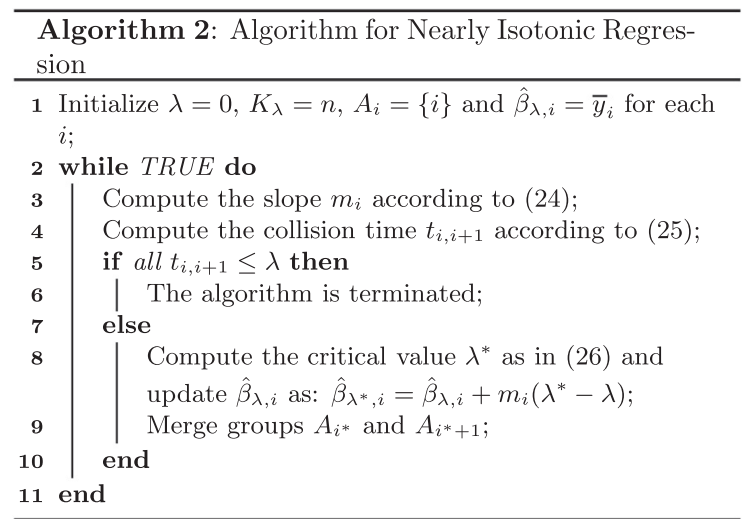

\subsection{Compute routing matrix}

Recall that the routing matrix $\boldsymbol{\Phi}$ in Bayesian Estimation is a binary matrix that randomly picks $L$ active nodes from the set of $N$ sensors. In Isotonic Regression, the routing matrix is not a random matrix; it chooses active nodes based on the estimation errors of each pair of sensor nodes.

Define $\epsilon_{X, Y}$ as the estimation error at sensor $s_{Y}$ due to reconstruction of the readings at sensor $s_{X}$, as

$$
\begin{aligned}
\epsilon_{X, Y} & =\frac{1}{K} \sum_{k=1}^{K}\left(y_{k}-f\left(x_{k}\right)\right)^{2} \text { for } X \neq Y \text { and } X, Y \\
& \in\{1, \ldots, N\}
\end{aligned}
$$

where $y_{k}$ is a reading from $s_{Y}$ and $f\left(x_{k}\right)$ is the estimate of $y_{k}$ based on the reading of $s_{X}$. We assume $\epsilon_{X, X}=0$ for all nodes. For the $N$ sensor nodes, we can construct an $N \times N$ matrix $\boldsymbol{\Psi}$ whose element is the estimation error of each pair of sensors. We compare the elements of matrix $\boldsymbol{\Psi}$ with a preset threshold $\xi$ and choose the pair whose corresponding estimation error is not greater than $\xi$.

The algorithm for finding the routing matrix $\boldsymbol{\Phi}$ is presented in Algorithm 3. Define $\Omega$ as the set of all sensor nodes and $\Omega_{1}$ as the set of active sensor nodes. In the beginning of the algorithm, we let $\Omega=\{1, \cdots, N\}$ and $\Omega_{1}=\varnothing$. In Lines 3-12, we find the minimum estimation error $\epsilon_{i^{*}, j^{*}}$ and compare it with the threshold $\xi$. If it is not greater than $\xi$, we remove $i^{*}$ from $\Omega$ and move $j^{*}$ from $\Omega$ to $\Omega_{1}$. These lines are executed until all nodes in $\Omega$ are removed. Once the algorithm is terminated, the set $\Omega_{1}$ is the set of nodes we would like to turn on. The nodes not in $\Omega_{1}$ is scheduled to be switched off. The complexity of this algorithm is $\mathcal{O}\left(N^{3}\right)$ 


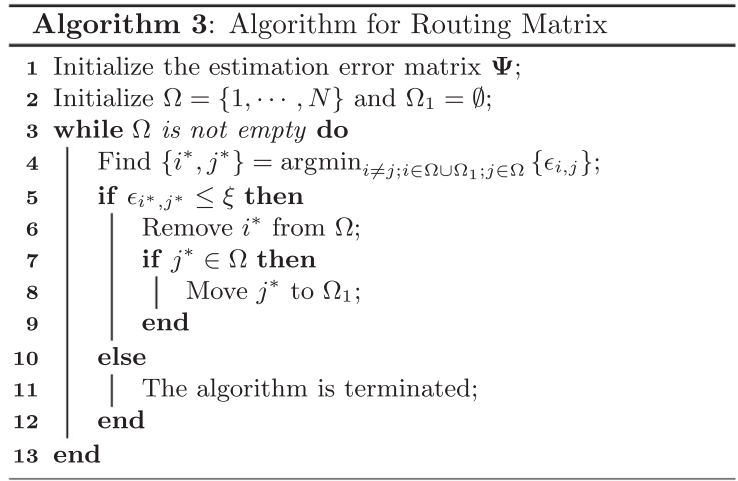

\section{Polynomial regression approaches}

If we treat the readings from $s_{X}$ as predictor variables and those from $s_{Y}$ as response variables, it is natural to use polynomial regression to estimate the readings of $s_{Y}$ from those of $s_{X}$. In this section, we examine how to use Linear Regression (LR) and Quadratic Regression (QR) in CS.

\subsection{Linear regression}

As in [22], we also adopt the simple LR method in CS. The reading of sensor node $s_{Y}$ can be estimated from the reading of sensor node $s_{X}$ with an LR model as follows.

$\hat{y}_{k}=\alpha_{0}+\alpha_{1} \cdot x_{k}, \quad$ for $k=1, \ldots, K$,

where $\alpha_{0}$ and $\alpha_{1}$ are the intercept and slope of the linear regression model.

Once $\hat{y}_{k}$ is obtained, the estimation error $\epsilon_{X, Y}$ and estimation error matrix $\boldsymbol{\Psi}$ can be computed as shown in Section 4.3. Similarly, the routing matrix for LR is obtained as described in Algorithm 3.

\subsection{Quadratic regression}

To compare with the LR method, we also choose a QR model as follows.

$\hat{y}_{k}=\alpha_{0}+\alpha_{1} \cdot x_{k}+\alpha_{2} \cdot\left(x_{k}\right)^{2}, \quad$ for $k=1, \ldots, K$,

where $\alpha_{2}$ is the coefficient of the second order term of $x_{k}$. The estimation error and routing matrix are obtained as in the case of LR. The complexity of LR and QR is $O(1)$ because the coefficients can be computed with explicit mathematic expressions. The rest of this method is the same as that in the LR method.

\section{Performance evaluation}

In this section, we evaluate the performance of all the methods with trace-driven simulations. The simulation code was written in MATLAB. We use the traces from the sensors deployed in an outdoor environment [28]. There are $N=16$ sensors that measure the illumination in a field. The range of the measurements is from 0 to 255 and they are collected from time $k=1$ to 150 . The scatter plot of the measured data is presented in Fig. 2.

We define Mean Square Error (MSE) as:

MSE $=\frac{1}{K \times N} \sum_{k=1}^{K} \sum_{i=1}^{N}\left(\hat{x}_{k}^{i}-x_{k}^{i}\right)^{2}$,

where $x_{k}^{i}$ is the reading at the $i$ th sensor at time $k$ and $\hat{x}_{k}^{i}$ the estimate of $x_{k}^{i}$. Note that $\hat{x}_{k}^{i}=x_{k}^{i}$ if the $i$ th sensor is active. MSE is computed for $k=51$ to 150 . At each time $k$, the previous 50 data vectors are used for parameter estimation.

We first compare the performance of the $B E$ approaches. We name the BE approaches with Gaussian and Laplace assumptions as BEG and BEL, respectively. Our proposed improvements are named IBEG and IBEL. In the simulation, we increase the number of active nodes from 5 to 9. Four curves of the BE approaches are plotted in Fig. 3. As expected, the more active nodes, the more accurate the prediction and the smaller the estimation error. We find our proposed enhancements yield lower MSE than those of the original schemes. IBEL has the best performance among the four BE-based methods, indicating that Laplace assumption is preferred in our proposed method.

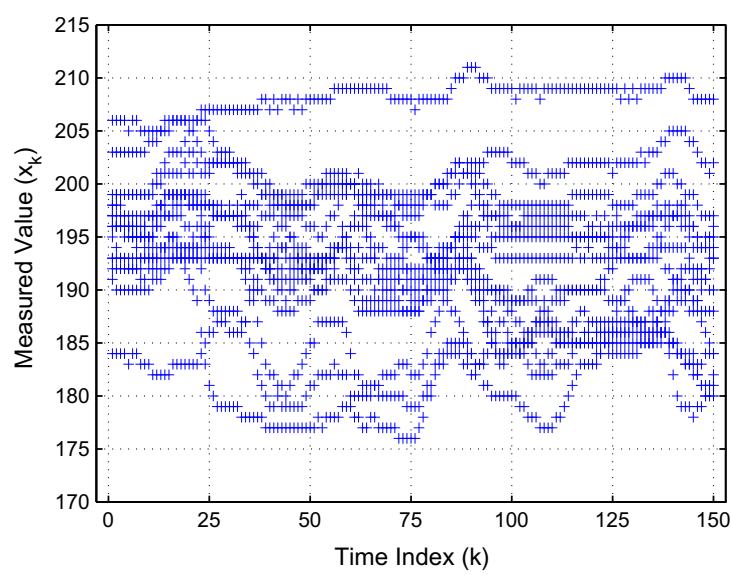

Fig. 2. The scatter plot of the trace data.

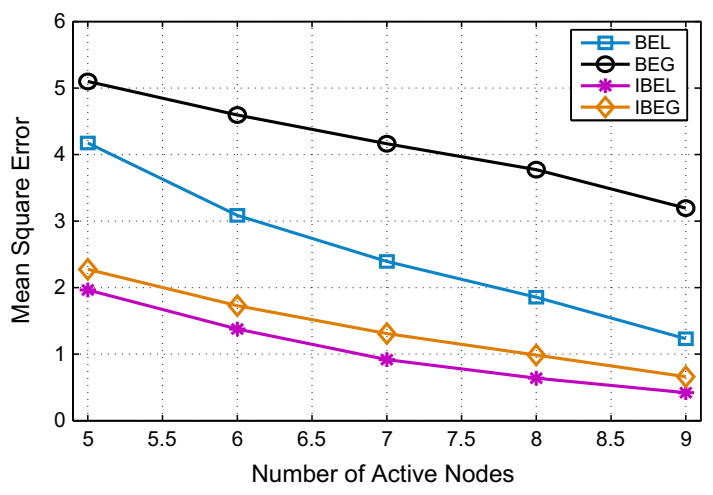

Fig. 3. Comparison of the BE approaches. 

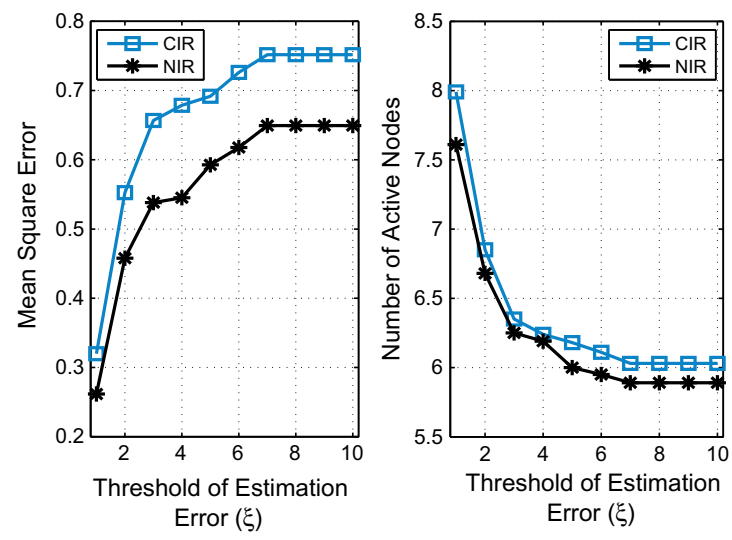

Fig. 4. Comparison of the IR approaches.
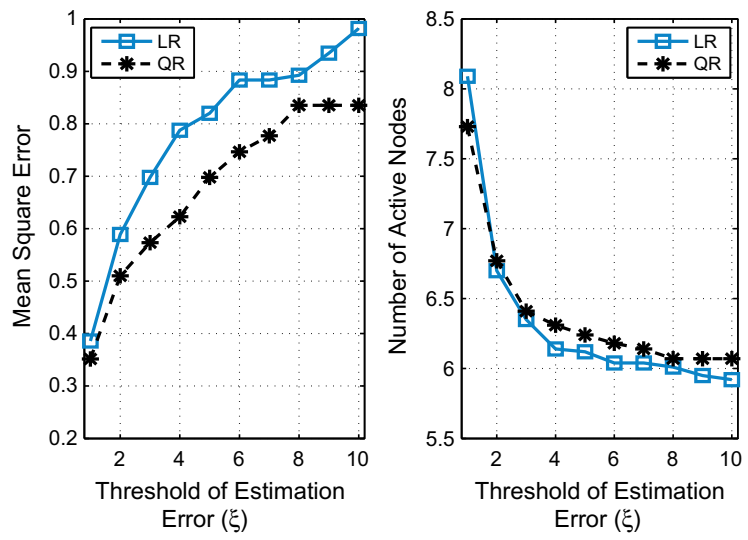

Fig. 5. Comparison of the PR approaches.

Next, we investigate the impact of estimation error threshold $\xi$ on CIR and NIR. In Fig. 4, we increase $\xi$ from 1 to 10 and plot MSE and the number of active nodes. Intuitively, a larger $\xi$ means a larger tolerance on estimation error, allowing more nodes to be turned off to save more energy. It can be seen from the figure that the MSE of NIR is up to 0.13 lower than that of CIR. Furthermore, we find NIR requires less active nodes than CIR. Therefore, NIR is superior to CIR with respect to both estimation error and energy savings. We thus choose it to compare with other methods in the following simulations.

In Fig. 5, we examine the impact of estimation error threshold $\xi$ on LR and QR. We increase $\xi$ from 1 to 10 and plot MSE and the number of active nodes in the figure. We find that although the MSE of QR is lower than that of $\mathrm{LR}, \mathrm{QR}$ requires more active nodes and thus consumes more power for computation and communication. Compared with QR, LR may be a sufficiently good choice for CS.

Finally, we choose one method from each of the three CS classes discussed above and compare their performance. Specifically, we choose IBEL, NIR and LR in this simulation. For each threshold of estimation error in Figs. 4

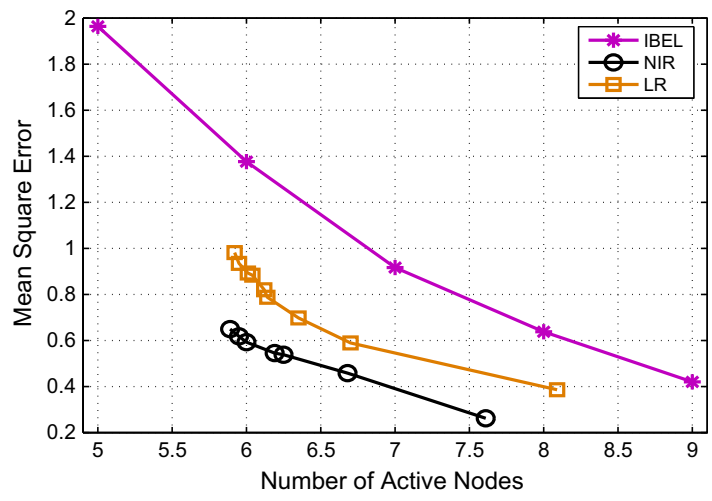

Fig. 6. Comparison of three approaches.

and 5, the $Y$-axis value is the corresponding MSE and the $X$-axis value is the corresponding number of active nodes. Three curves are plotted in Fig. 6 for the three selected schemes, respectively. Although IBEL requires as few as five active nodes, its MSE is very high. Among these three methods, it is obvious that NIR achieves the best performance because it has smallest MSE and requires the minimum number of active nodes.

\section{Conclusion}

In this paper, we investigated three classes of CS approaches with focus on balancing the trade-off between energy efficiency and estimation error in a WSN environment. We first introduced Bayesian Estimation based approaches and propose an enhanced scheme by scaling the variance of projected random variables. We then adopted NIR to improve the performance of CIR. LR and QR approaches are also examined for comparison purpose. We compared the three classes of CS approaches with tracedriven simulations. Our simulation results showed that NIR is the best choice among all the CS approaches considered in this paper.

\section{Acknowledgments}

This work is supported in part by the US National Science Foundation (NSF) under Grants CNS-0953513, and through the NSF Broadband Wireless Access and Applications (BWAC) Center site at Auburn University.

\section{References}

[1] D. Hu, S. Mao, N. Billor, On balancing energy efficiency and estimation error in compressed sensing, in: Proc. IEEE GLOBECOM 2012, Anaheim, CA, 2012, pp. 1-6.

[2] E.J. Candés, M.B. Wakin, An introduction to compressive sampling, IEEE Signal Process. Mag. 25 (2) (2008) 21-30.

[3] I.F. Akyildiz, B.F. Lo, R. Balakrishnan, Cooperative spectrum sensing in cognitive radio networks: a survey, IEEE Commun. Mag. 4 (1) (2011) 40-62.

[4] I.F. Akyildiz, W.-Y. Lee, M.C. Vuran, S. Mohanty, A survey on spectrum management in cognitive radio networks, IEEE Commun. Mag. 46(4) (2008) 40-48. 
[5] Q. Zhao, B. Sadler, A survey of dynamic spectrum access, IEEE Signal Process. Mag. 24 (3) (2007) 79-89.

[6] Y. Zhao, S. Mao, J.O. Neel, J.H. Reed, Performance evaluation of cognitive radios: metrics, utility functions, and methodology, The Proceedings of the IEEE. 97(4) (2009) 642-659.

[7] D. Hu, S. Mao, Cooperative relay with interference alignment for video over cognitive radio networks, in: Proc. IEEE INFOCOM 2012 Orlando, FL, 2012, pp. 2014-2022.

[8] I.F. Akyildiz, W. Su, Y. Sankarasubramaniam, E. Cayirci, A survey on sensor networks, IEEE Commun. Mag. 40 (8) (2002) 102-114.

[9] I.F. Akyildiz, I.H. Kasimoglu, Wireless sensor and actor networks: research challenges, Elsevier Ad Hoc Networks Journal 2 (4) (2004) 351-367.

[10] F. Ye, G. Zhong, J. Cheng, S. Lu, L. Zhang, PEAS: a robust energy conserving protocol for long-lived sensor networks, in: Proc. 23rd Int. Distributed Computing Systems Conf., 2003, pp. 28-37, http:// dx.doi.org/10.1109/ICDCS.2003.1203449.

[11] H. Ling, T. Znati, Energy efficient adaptive sensing for dynamic coverage in wireless sensor networks, in: Proc. IEEE WCNC'09, 2009, pp. 1-6, http://dx.doi.org/10.1109/WCNC.2009.4917708.

[12] C.-F. Hsin, M. Liu, Network coverage using low duty-cycled sensors: random and coordinated sleep algorithms, in: Proc. ACM IPSN'04 2004, pp. 433-442, http://dx.doi.org/10.1109/IPSN.2004.1307365.

[13] X. Wang, G. Xing, Y. Zhang, C. Lu, R. Pless, C. Gill, Integrated coverage and connectivity configuration in wireless sensor networks, in: Proc. ACM SenSys'03, Los Angeles, CA, 2003, pp. 28-39.

[14] T. Yan, T. He, J.A. Stankovic, Differentiated surveillance for sensor networks, in: Proc. ACM SenSys'03, Los Angeles, CA, 2003, pp. 51-62.

[15] T. He, S. Krishnamurthy, J.A. Stankovic, T. Abdelzaher, L. Luo, R Stoleru, T. Yan, L. Gu, J. Hui, B. Krogh, Energy-efficient surveillance system using wireless sensor networks, in: Proc. ACM MobiSys'04 Boston, MA, 2004, pp. 270-283.

[16] Q. Cao, T. Abdelzaher, T. He, J. Stankovic, Towards optimal sleep scheduling in sensor networks for rare-event detection, in: Proc. ACM IPSN'05, Los Angeles, CA, 2005, pp. 20-27, http://dx.doi.org/ 10.1109/IPSN.2005.1440887.

[17] P. Wang, R. Dai, I.F. Akyildiz, Collaborative data compression using clustered source coding for wireless multimedia sensor networks, in: Proc. IEEE INFOCOM 2010, San Diego, CA, 2010, pp. 1-9.

[18] D. Donoho, Compressed sensing, IEEE Trans. Inform. Theory 52 (4) (2006) 4036-4048.

[19] E. Candes, T. Tao, Near optimal signal recovery from random projections: universal encoding strategies?, IEEE Trans Inform. Theory 52 (12) (2006) 5406-5425.

[20] J.R.E. Candes, T. Tao, Robust uncertainty principles: exact signal reconstruction from highly incomplete frequency information, IEEE Trans. Inform. Theory 52 (2) (2006) 489-509.

[21] F. Koushanfar, N. Taft, M. Potkonjak, Sleeping coordination for comprehensive sensing using isotonic regression and domatic partitions, in: Proc. IEEE INFOCOM'06, Barcelona, Spain, 2006, pp. 1-13, http://dx.doi.org/10.1109/INFOCOM.2006.276.

[22] G. Ollos, R. Vida, Adaptive regression algorithm for distributed dynamic clustering in wireless sensor networks, in: Proc. 2nd IFIP Wireless Days (WD), Paris, France, 2009, pp. 1-5, http://dx.doi.org/ 10.1109/WD.2009.5449687.

[23] R. Masiero, G. Quer, D. Munaretto, M. Rossi, J. Widmer, M. Zorzi, Data acquisition through joint compressive sensing and principal component analysis, in: Proc. IEEE Globecom 2009, Honolulu, HI, 2009.

[24] R. Masiero, G. Quer, M. Rossi, M. Zorzi, A Bayesian analysis of compressive sensing data recovery in wireless sensor networks, in: Proc. 2009 Int. Conf. Ultra Modern Telecommun. Workshops, St.Petersburg, Russia, 2009, pp. 1-6.

[25] M. Grant, S. Boyd, Graph implementations for nonsmooth convex programs, in: V. Blondel, S. Boyd, H. Kimura (Eds.), Recent Advances in Learning and Control, Lecture Notes in Control and Information Sciences, Springer-Verlag Limited, 2008, pp. 95-110.

[26] M. Grant, S. Boyd, CVX: Matlab Software for Disciplined Convex Programming, Version 1.21, April 2011. <http://cvxr.com/cvx>.

[27] R. Tibshirani, H. Hoefling, R. Tibshirani, Nearly-isotonic regression, Technometrics 53 (1) (2010) 54-61.

[28] Wireless Sensor Network Illumination Data Trace. <http:// lightning.med.monash.edu/Illumination.zip>.

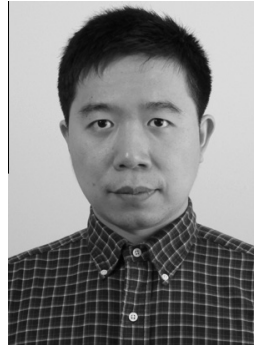

Donglin $\mathrm{Hu}$ received the M.S. degree from Tsinghua University, Beijing, China, in 2007 and the B.S. degree from Nanjing University of Posts and Telecommunications, Nanjing, China in 2004, respectively, all in electrical engineering. He received the M.S. degree in Probability and Statistics from Auburn University, Auburn, AL, in 2011, and the Ph.D. degree in Electrical and Computer Engineering from Auburn University in 2012. Currently he is a postdoctoral research fellow in the Department of Electrical and Computer Engineering at Auburn University. His research interests include cognitive radio networks, femtocell networks, network modeling, cross-layer design, performance analysis, and algorithm optimization for wireless networks and multimedia communications.

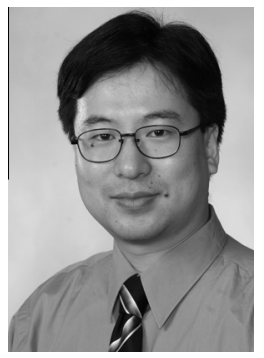

Shiwen Mao received Ph.D. in electrical and computer engineering from Polytechnic University, Brooklyn, NY, USA (now Polytechnic Institute of New York University) in 2004. He was a research staff member with IBM China Research Lab from 1997 to 1998 . He was a Postdoctoral Research Fellow/Research Scientist in the Bradley Department of Electrical and Computer Engineering at Virginia Polytechnic Institute and State University (Virginia Tech), Blacksburg, VA, USA from 2003 to 2006. Currently, he is the McWane Associate Professor in the Department of Electrical and Computer Engineering, Auburn University, Auburn, AL, USA. His research interests include crosslayer optimization of wireless networks and multimedia communications, with current focus on cognitive radios, femtocell networks, $60 \mathrm{GHz}$ mmWave networks, free space optical networks, and smart grid. He is on the Editorial Board of IEEE Transactions on Wireless Communications, IEEE Communications Surveys and Tutorials, Elsevier Ad Hoc Networks Journal, Wiley International Journal of Communication Systems, and ICST Transactions on Mobile Communications and Applications. He is the Director of E-Letter of the Multimedia Communications Technical Committee (MMTC), IEEE Communications Society for 2012-2014. He is a coauthor of TCP/IP Essentials: A Lab-Based Approach (Cambridge University Press, 2004). He was awarded the McWane Endowed Professorship in the Samuel Ginn College of Engineering for the Department of Electrical and Computer Engineering, Auburn University in August 2012. He received the US National Science Foundation Faculty Early Career Development Award (CAREER) in 2010. He is a co-recipient of The 2004 IEEE Communications Society Leonard G. Abraham Prize in the Field of Communications Systems and The Best Paper Runner-up Award at The Fifth International Conference on Heterogeneous Networking for Quality, Reliability, Security and Robustness (QShine) in 2008. He was named 2012 Exemplary Editor of IEEE Communications Surveys and Tutorials. He also received Auburn Alumni Council Research Awards for ExcellenceJunior Award in 2011 and two Auburn Author Awards in 2011. Dr. Mao holds one US patent.

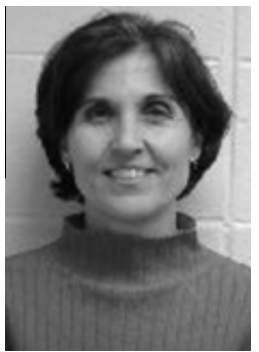

Nedret Billor received her Ph.D. from University of Sheffield, UK. She is a professor in the Department of Mathematics and Statistics, Auburn University, Auburn, AL, USA. Her research interests include Outlier detection in regression and multivariate data, Robust multivariate and functional data analysis. She has authored numerous publications in journals which have garnered over many citations. She is a fellow of the Royal Statistical Society, elected member of the International Statistical Institute. 


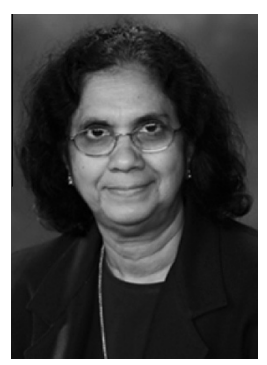

Prathima Agrawal is the Samuel Ginn distinguished professor of electrical and computer engineering and the director of the Wireless Engineering Research and Education Center, Auburn University, Auburn, Alabama. Prior to her present positions, she worked in Telcordia Technologies (formerly Bellcore), Morristown, New Jersey, and at AT\&T/Lucent Bell Laboratories, Murray Hill, New Jersey. She created and served as head of the Networked Computing Research Department in Murray Hill. She is widely published and holds 51 US patents. She is a Fellow of IEEE. She received the BE and ME degrees in electrical communication engineering from the Indian Institute of Sci- ence, Bangalore, India, and the $\mathrm{PhD}$ degree in electrical engineering from the University of Southern California in 1977. 